\title{
REFERENCES
}

1. L. Cesari, Asymptotic behavior and stability problems in ordinary differential equations, Springer-Verlag, Berlin, 1959.

2 A. Lyapunov, Problème genéral de la stabilité der movement, Toulouse Univ., Fac. Sci. Ann. (2) 9 (1907), 203-474. Reprinted by Princeton Univ. Press.

3. W. Magnus, and A. Shenitzer, Hill's equation. I. General theory, New York Univ., Inst. Math. Sci., Div. EM Res., Research Report No. BR-22 (1957).

4. H. Hochstadt, Asymptotic estimates for the Sturm-Liouville spectrum, New York Univ., Inst. Math. Sci., Div. EM Res., Research Report No. BR-36; to be published, Comm. Pure Appl. Math. (1961).

NEW YoRK UNIVERSITY

\section{COMPARISON THEOREMS FOR LINEAR DIFFERENTIAL EQUATIONS OF SECOND ORDER}

\section{WALTER LEIGHTON ${ }^{1}$}

1. In this paper we consider self-adjoint differential equations of the form

$$
\left[r(x) y^{\prime}\right]^{\prime}+p(x) y=0,
$$

where $r(x)$ and $p(x)$ are continuous and $r(x)>0$ on an interval $\alpha<x<\beta$. By rewriting a theorem in the calculus of variations in a form which emphasizes the behavior of solutions of the Euler-Jacobi equation rather than that of the functional which gives rise to it we are led to observe that the theorem provides a completely general comparison theorem for equations of the form (1). We show that the Sturm and Sturm-Picone ${ }^{2}$ theorems may be regarded as special cases of this theorem and incidentally provide in the process useful generalizations of these theorems.

We associate with (1) the functional

$$
I=\int_{a}^{b}\left(r u^{\prime 2}-p u^{2}\right) d x
$$

where the closed interval $[a, b] \subset(\alpha, \beta)$. If $u(x)$ and $r(x) u^{\prime}(x)$ are functions of class $C^{\prime}$ on $[a, b]$ and if $u(a)=u(b)=0$, we shall say that

Received by the editors April 24, 1961 and, in revised form, June 29, 1961.

1 Work on this paper was done while the author received support from the $\mathrm{Na}$ tional Science Foundation under grant NSF-G11741.

2 See, for example, Bocher [1, p. 53], Ince $[2$, p. 225]. 
$u(x)$ is admissible. An easy integration by parts then establishes the functional identity

$$
\begin{aligned}
I & =\left.r u u^{\prime}\right|_{a} ^{b}-\int_{a}^{b} u\left[\left(r u^{\prime}\right)^{\prime}+p u\right] d x \\
& =-\int_{a}^{b} u\left[\left(r u^{\prime}\right)^{\prime}+p u\right] d x=-J,
\end{aligned}
$$

where $u(x)$ is any admissible function.

We state the following theorem.

PRINCIPLE. If $y(x) \not \equiv 0$ is a solution of (1) such that $y(a)=0$, then $a$ necessary and sufficient condition that $y(c)=0$, where $a<c<b$, is that there exist an admissible function $u(x)$ for which $I<0$.

This theorem, which is usually stated in a different form, is wellknown. A useful form of the sufficiency condition may be stated as follows.

THEOREM 1. If $u(x)$ is an admissible function and if

$$
J=\int_{a}^{b} u(x)\left[\left(r(x) u^{\prime}(x)\right)^{\prime}+p(x) u(x)\right] d x>0
$$

a solution $y(x)$ of (1) which vanishes at $x=a$ has the property that $y(c)=0$, where $a<c<b$.

Note that $u(x)$ is not required to be different from zero on the interval $a<x<b$.

A simple but instructive example of an application of Theorem 1 is provided by setting $u(x)=\sin k x, r=1, p=m^{2}(m>k>0), a=0$, $b=\pi / k$. Then

$$
J=\int_{a}^{b} u\left[\left(r u^{\prime}\right)^{\prime}+p u\right] d x=\int_{0}^{\pi / k}\left(m^{2}-k^{2}\right) \sin ^{2} k x d x>0 .
$$

Accordingly, a solution $y(x)$ of

$$
y^{\prime \prime}+m^{2} y=0
$$

such that $y(0)=0$ must vanish on the interval $0<x<\pi / k$, as was to have been anticipated.

TheOREM 2. Let $r(x)$ and $r_{1}(x)$ be positive and $r(x), r_{1}(x), p(x)$, and $p_{1}(x)$ be continuous on the interval $\alpha<x<\beta$ and consider the differential equations 


$$
\begin{gathered}
{\left[r_{1}(x) u^{\prime}\right]^{\prime}+p_{1}(x) u=0,} \\
{\left[r(x) y^{\prime}\right]^{\prime}+p(x) y=0 .}
\end{gathered}
$$

If there exists an admissible function $u(x)$ such that

$$
\int_{a}^{b}\left[\left(r_{1}-r\right) u^{\prime 2}+\left(p-p_{1}\right) u^{2}\right] d x>\int_{a}^{b}\left(r_{1} u^{\prime 2}-p_{1} u^{2}\right) d x
$$

a solution $y(x)$ of (4) such that $y(a)=0$ has the property that $y(c)=0$, where $a<c<b$.

The proof of the theorem is very simple. From the above Principle it follows that if $u(x)$ is any admissible function for which

$$
\int_{a}^{b} p u^{2} d x>\int_{a}^{b} r u^{\prime 2} d x
$$

the conclusion of the theorem follows. But (6) is formally equivalent to (5), where $r_{1}(x)$ and $p_{1}(x)$ are quite arbitrary functions of the type described in the theorem.

The proof of the theorem is complete.

Note that if $u(x)$ is a solution of (3) such that $u(a)=u(b)=0$, then $u(x)$ is admissible and the right-hand member of (5), after an integration by parts of its first term, is readily seen to be zero. The following corollary is then immediate.

CoROllary 1. If $u(x)$ is a solution of (3) such that $u(a)=u(b)=0$, a solution $y(x)$ of (4) such that $y(a)=0$ will have the property that $y(c)=0(a<c<b)$, provided

$$
\int_{a}^{b}\left[\left(r_{1}-r\right) u^{\prime 2}+\left(p-p_{1}\right) u^{2}\right] d x>0 .
$$

This corollary is a generalization of the Sturm-Picone Theorem, for a corollary of this corollary yields the following version of that theorem:

COROLlary 2. The conclusion of Theorem 2 holds if

$$
r(x) \leqq r_{1}(x), \text { and } p(x) \geqq p_{1}(x) \quad(a \leqq x \leqq b),
$$

provided at least one of the conditions (8) holds as an actual inequality at some point of the interval $[a, b]$.

The proof is trivial.

Setting $r_{1}(x)=r(x)$ in Corollary 1 yields the following result.

CoROLlaRY 3. If $r_{1}(x)=r(x)$, the conclusion of Theorem 2 holds pro- 
vided there exists an admissible solution $u(x)$ of (3) for which

$$
\int_{a}^{b}\left(p-p_{1}\right) u^{2}(x) d x>0 .
$$

It will be seen that the ordinary Sturm comparison theorem is an immediate consequence of Corollary 3.

Theorem 2, incidentally, extends and clarifies a result due to the author [3].

In the following example of the use of Theorem 2 note that we take $p_{1}<0$ so that no nontrivial solution of (3) can be admissible, but, nevertheless, we have a valid comparison theorem.

EXAmple. Let $r_{1}=2, p_{1}=-2, r=1$, and $p$ be a constant. Then equations (3) and (4) become, respectively,

$$
\begin{aligned}
u^{\prime \prime}-u & =0, \\
y^{\prime \prime}+p y & =0 .
\end{aligned}
$$

We take $u(x)=\sin x, a=0, b=\pi[u(x)$ is not a solution of (3)']. Here (5) becomes

$$
\int_{0}^{\pi}\left[\cos ^{2} x+(p+2) \sin ^{2} x\right] d x>\int_{0}^{\pi} 2 d x
$$

and this result will be seen to hold provided $p>1$. That is, if $p$ is a constant $>1$, a nontrivial solution $y(x)$ of $(4)^{\prime}$ such that $y(0)=0$ will vanish again on the interval $0<x<\pi$-which is well-known, of course.

The following example is less trivial.

EXAMPLE. Consider the differential system

$$
\begin{aligned}
y^{\prime \prime}+(x+1-k) y & =0 \\
y(0) & =0 .
\end{aligned} \quad(0<k<\pi / 2),
$$

Here, $r=1, p=x+1-k$. We compare a nonnull solution $\bar{y}(x)$ of this system with the solution $\sin x$ of the system

$$
y^{\prime \prime}+y=0, \quad y(0)=0 \text {. }
$$

Using (9) we have

$$
J=\int_{0}^{\pi}(x-k) \sin ^{2} x d x=\pi(\pi / 2-k)>0 .
$$

Accordingly, $\bar{y}(c)=0$, where $0<c<\pi$. It will be noted that neither the Sturm Theorem nor the Sturm-Picone Theorem applies.

A somewhat more general result than the last corollary may be 
obtained as follows. Let $u(x)$ be a solution of equation (3) such that $u(a)=u(b)=0$. It follows from Theorem 1 that if $r(x)$ and $r_{1}(x)$ are of class $C^{\prime}$ on $(\alpha, \beta)$, the conclusion of Theorem 1 (or 2) will follow provided $J>0$. If in $J$ we write $r u^{\prime}$ in the form $r\left(r_{1} u^{\prime}\right) / r_{1}$, the condition $J>0$ may be readily written in the form

$$
\int_{a}^{b} u\left[\left(p-\frac{r}{r_{1}} p_{1}\right) u+r_{1} u^{\prime}\left(\frac{r}{r_{1}}\right)^{\prime}\right] d x>0 .
$$

Thus, for example, if $r / r_{1}=$ constant this condition becomes

$$
\int_{a}^{b}\left(p-\frac{r}{r_{1}} p_{1}\right) u^{2} d x>0 .
$$

Another immediate consequence of the Principle given above is the following result. It will be seen to be equivalent to the necessity of the Jacobi condition in the calculus of variations.

THEOREM 3. If $y(x) \not \equiv 0$ is a solution of equation (4) such that $y(a)$ $=y(c)=0(a<c<b)$, there exists an admissible function $u(x)$ for which $I<0$ (or, $J>0$ ).

2. The typical proof of Theorem 3 involves the construction of a function of class $D^{\prime}$ ( $C^{\prime}$ except for a finite number of corners). In this section we provide a function of class $C^{\prime}$ which in a large class of cases gives $I$ a negative value when the (open) interval contains a conjugate point. Not only is this intrinsically interesting but it also leads to a novel kind of disconjugacy theorem for equations of the type (1).

To simplify matters we consider, without loss in generality, the functional

$$
I(u)=\int_{0}^{b c}\left[r(x) u^{\prime 2}-p(x) u^{2}\right] d x \quad(b>1),
$$

where $r(x)>0$ and $r(x)$ and $p(x)$ are continuous on an open interval $(\alpha, \beta)$ which contains the interval $[0, b c]$. Associated with $(10)$ is its Euler-Jacobi equation

$$
\left[r(x) u^{\prime}\right]^{\prime}+P(x) u=0 .
$$

We first deal with the following result.

THEOREM 4. If a nonnull solution $u(x)$ of (11) has the property that $u(0)=u(c)=0 \quad(0<c<b c)$, the function $v(x)=u(x / b) \quad(b>1)$ makes $I \leqq 0$, at least if $b-1$ is sufficiently small.

To prove the theorem we compute $I(v)$ obtaining 


$$
\begin{aligned}
I(v) & =\int_{0}^{b c}\left[r(x) v^{\prime 2}(x)-p(x) v^{2}(x)\right] d x \\
& =\int_{0}^{b c}\left[\frac{1}{b^{2}} r(x) u^{\prime 2}\left(\frac{x}{b}\right)-p(x) u^{2}\left(\frac{x}{b}\right)\right] d x .
\end{aligned}
$$

Under the transformation $x=b t$ we have

$$
I(v)=b \int_{0}^{c}\left[\frac{1}{b^{2}} r(b t) u^{\prime 2}(t)-p(b t) u^{2}(t)\right] d t .
$$

It will be sufficient to show that

$$
I_{1}=\int_{0}^{c}\left[r(b t) u^{\prime 2}(t)-b^{2} p(b t) u^{2}(t)\right] d t \leqq 0 .
$$

Note that $w(x)=u(b x)$ is a solution of the differential equation

$$
\left[r(b x) w^{\prime}\right]^{\prime}+b^{2} p(b x) w=0,
$$

and that $w(c / b)=0$.

It will be convenient to add and subtract the tern $r(t) u^{\prime 2}(t)$ under the integral in (12). One obtains, after an integration by parts,

$$
I_{1}=\int_{0}^{c}\left\{[r(b t)-r(t)] u^{\prime 2}(t)+\left[p(t)-b^{2} p(b t)\right] u^{2}(t)\right\} d t
$$

Suppose $I_{1}=k^{2}>0$, and consider the functional

$$
J=\int_{0}^{c / b}\left\{[r(b t)-r(t)] w^{\prime 2}(t)+\left[p(t)-b^{2} p(b t)\right] w^{2}(t)\right\} d t .
$$

We set $w^{\prime}(0)=u^{\prime}(0)$ (this amounts to setting $w(x)=m u(b x)(m=1 / b)$ ). Then, as $b \rightarrow 1, w(x) \rightarrow u(x), w^{\prime}(x) \rightarrow u^{\prime}(x)$, and, accordingly, for $b-1$ sufficiently small $J$ will be positive. It would then follow from Corollary 1 of Theorem 2 that the first positive zero $x=c$ of $u(x)$ would precede the zero $x=c / b$ of $w(x)$, which is absurd since $b>1$. From this contradiction we infer that $I_{1} \leqq 0$, and the proof of Theorem 4 is complete.

It is readily seen that Theorem 4 is not valid for all $b>1$, for consider the functional

$$
J=\int_{0}^{b \sqrt{6} / 2}\left[y^{\prime 2}-\left(7-x^{2}\right) y^{2}\right] d x .
$$

The associated Euler-Jacobi equation is 


$$
y^{\prime \prime}+\left(7-x^{2}\right) y=0
$$

and a solution of this is

$$
y=e^{-x^{2} / 2}\left(2 x^{3}-3 x\right)
$$

The solution $v(x)$ becomes

$$
e^{-x^{2} / 2 b^{2}}\left(\frac{2 x^{3}}{b^{3}}-\frac{3 x}{b}\right)
$$

and

$$
\begin{aligned}
J(v) & =-\int_{0}^{b \sqrt{6} / 2} v\left[v^{\prime \prime}+\left(7-x^{2}\right) v\right] d x \\
& =\frac{b^{2}-1}{b} \int_{0}^{\sqrt{ } / 2} y^{2}(t)\left[\left(b^{2}+1\right) t^{2}-7\right] d t \quad(x=b t, b>1) .
\end{aligned}
$$

It is clear that $b$ can be taken large enough to make $J(v)>0$.

But more is suggested by (14). We obtain the following theorem.

THEOREM 5. If the functions $r(x)$ and $p(x)$ in the differential equation

$$
\left[r(x) y^{\prime}\right]^{\prime}+p(x) y=0
$$

are continuous on an interval $a \leqq x<d(a>0)$ and if for every number $b$ on some interval $1<b<1+c(c>0)$

$$
r(b x) \geqq r(x), \quad p(x) \geqq b^{2} p(b x) \quad(a \leqq x<d),{ }^{8}
$$

with strict inequality holding in one of these conditions, a solution $u(x)$ of (15) such that $u(a)=0, u^{\prime}(a) \neq 0$ cannot vanish more than once on the interval $a<x<d$.

To prove the theorem note that conditions (16) insure that the functional in (14) will be positive over any interval contained in the interval $a \leqq x<d$ for any admissible function $u(x)$. Suppose that $u\left(c_{1}\right)=u\left(c_{2}\right)=0$, where $a<c_{1}<c_{2}<d$. By Corollary 1 of Theorem 2 a nonnull solution $w_{1}(x)$ of (13) such that $w_{1}\left(c_{1}\right)=0$ will be different from zero when $c_{1}<x \leqq c_{2}+\epsilon$, where $\epsilon$ is a small positive number. There will then exist a solution $w_{2}(x)$ of (13) with the property that $w_{2}(x)>0$ for $c_{1} \leqq x \leqq c_{2}+\epsilon_{1}$, where $\epsilon_{1}$ is a small positive number, for each $b>1$, but near 1 . Next, observe that we may choose a solution

\footnotetext{
${ }^{8}$ The referee made the interesting observation that the second condition (16) cannot be satisfied if $a=0$. He observed that an easy iteration of this condition leads to $p\left(x_{0} / b^{k}\right) \geqq b^{2 k} p\left(x_{0}\right), k=1,2, \cdots$, where $x=x_{0}$ is any point of the interval $a<x<d$; hence, $p(x)$ cannot be continuous at $x=0$.
} 
$u_{1}(x)$ of (15) with the property that $u_{1}\left(k_{1}\right)=u_{1}\left(k_{2}\right)=0$, where $c_{1}<k_{1}$, $c_{2}<k_{2}<c_{2}+\epsilon_{1}$. Recall that for each $b$ the function $w_{3}(x)=u_{1}(b x)$ is a solution of (13). By taking $b>1$, but sufficiently near $1, w_{3}(x)$, which has zeros at $k_{1} / r$ and $k_{2} / b$, can be taken to have the first of these zeros to the right of $x=c_{1}$, while the second will lie to the left of $x=k_{1}$. But this choice of $b$ for $w_{2}(x)$ contradicts the Sturm separation theorem.

From th:s contradiction we infer the truth of the theorem.

The theorem is sharp, for the following example exhibits a solution $u(x)$ of the type described in Theorem 5 which has one zero on the interval $a<x<d$.

EXAmple. The coefficients of the differential equation

$$
\left(\frac{x^{2}}{2 \pi a} y^{\prime}\right)^{\prime}+\frac{2 \pi a}{x^{2}} y=0 \quad(a>0)
$$

clearly satisfy condition (16) for all $b>1$. A solution $u(x)$ is

$$
\sin \left[2 \pi a\left(\frac{1}{a}-\frac{1}{x}\right)\right],
$$

which vanishes at $x=a$ and at $x=2 a$. This function has no zeros larger than $2 a$. A corresponding $w(x)$ is

$$
\sin \left[\frac{2 \pi a}{b}\left(\frac{1}{a}-\frac{1}{x}\right)\right] .
$$

Note that $w(x)=0$ when $x=a$ and $x=2 a /(2-b)$, when $b<2$; however, $w(x) \neq 0$ for $x>a$, if $b \geqq 2$.

\section{BIBLIOGRAPHY}

1. Maxime Bocher, Lȩ̧ons sur les methodes de Sturm, Gauthier-Villars, Paris, 1917.

2. E. L. Ince, Ordinary differential equations, Longmans, London, 1927.

3. Walter Leighton, $\boldsymbol{A}$ substitute for the Picone formula, Bull. Amer. Math. Soc. 55 (1949), 325-328.

4. Marston Morse, The calculus of variations in the large, Amer. Math. Soc. Colloq. Publ. Vol. 18, Amer. Math. Soc., New York, 1934.

WESTERN RESERVE UNIVERSITY 